\title{
Atomic Scale Structure and Reduction of Cerium Oxide at the Interface with Platinum
}

\author{
By Paola Luches, * Livia Giordano, Vincenzo Grillo, Gian Carlo Gazzadi, \\ Stefano Prada, Marco Campanini, Giovanni Bertoni, Cesar Magen, \\ Federico Pagliuca, Gianfranco Pacchioni, and Sergio Valeri
}

Keywords: DFT calculations, metal-oxide interaction, scanning transmission electron microscopy, STEM-EELS

\begin{abstract}
A detailed atomic scale description of the interfaces between cerium oxide and metals is necessary for a clear understanding of interfacial interactions, and it may open the way to the optimization of the properties of the combined material in view of its applications. In this study the interface between cerium oxide epitaxial films and the (111) surface of platinum is studied by aberration-corrected scanning transmission electron microscopy and by ab initio density functional theory calculations. Local modifications of the registry between the cerium oxide and platinum lattices and the occurrence of nanometric platinum islands at the interface are detected by high-angle annular dark field imaging. Moreover, the preferential adsorption sites of $\mathrm{Ce}$ and $\mathrm{O}$ atoms at the interface are determined by comparison of the images acquired in annular bright-field mode and the results of density functional theory calculations. The presence of a non-negligible concentration of $\mathrm{Ce}^{3+}$ ions at the interface is detected by electron energy loss spectroscopy and it is rationalized in terms of interfacial charge transfer as evidenced by the calculations. This work provides an insight into the atomic and electronic structure of the technologically relevant $\mathrm{CeO}_{2} / \mathrm{Pt}$ interface and it helps to clarify the properties of the combined material.
\end{abstract}

\section{Introduction}

Materials that combine metal nanoparticles and cerium oxide have shown to be very active in a number of catalytic reactions,

Dr. P. Luches, Dr. V. Grillo, Dr. G. C. Gazzadi, Dr. F. Pagliuca, Prof. S. Valeri, Istituto Nanoscienze, Consiglio Nazionale delle Ricerche, Via G. Campi 213/a, 41121 Modena, Italy

Dr. L. Giordano, Dr. S. Prada, Prof. G. Pacchioni, Dipartimento di Scienza dei Materiali, Università di Milano-Bicocca, Via R. Cozzi 5320125 Milano, Italy

Dr. V. Grillo, Dr. M. Campanini, Dr. G. Bertoni, Istituto Materiali per Elettronica e Magnetismo, Consiglio Nazionale delle Ricerche, Parco Area Delle Scienze 37/A 43124 Parma, Italy

Dr. C. Magen, Laboratorio de Microscopías Avanzadas (LMA), Instituto de Nanociencia de Aragón (INA)—ARAID, University of Zaragoza 50018 Zaragoza, Spain

Dr. C. Magen, Departamento de Fisica de la Materia

Condensada, University of Zaragoza 50018 Zaragoza, Spain Dr. F. Pagliuca, Prof. S. Valeri, Dipartimento di Scienze Fisiche Informatiche e Matematiche, Università di Modena e Reggio Emilia, Via G. Campi 213/a 41121 Modena, Italy Correspondence to: Dr. P. Luches (E-mail: paola.luches@unimore.it) 10.1002/admi.201500375 


\section{ADVANCED INTERFACES}

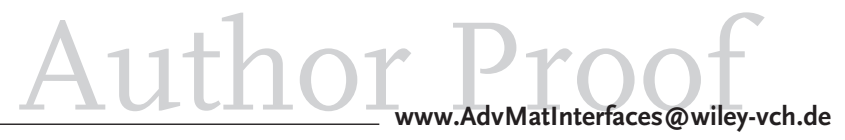

Since the (111) surface is the most stable one for cerium oxide ${ }^{[4]}$ a large number of studies have focused on the growth and properties of model systems in the form of $\mathrm{CeO}_{2}$ films with (111) orientation supported on metal surfaces with threefold symmetry, like (111) fcc and (0001) hcp metal surfaces. ${ }^{[5-12]}$ In spite of the significant lattice parameter mismatch between most metal surfaces with threefold symmetry and the $\mathrm{CeO}_{2}(111)$ surface $(30 \%-40 \%)$, good quality epitaxial cerium oxide films with the fluorite structure and atomically flat terraces have been obtained. ${ }^{[7-12]}$ Interesting properties of metal/cerium oxide interfaces are evident when the oxide film is ultrathin, i.e., below $\approx 1 \mathrm{~nm}$ thickness, where metastable phases with peculiar properties have been stabilized. ${ }^{[13-15]}$ For example, Castellarin-Cudia et al. ${ }^{[13]}$ showed that reduced ultrathin ceria films on $\mathrm{Rh}(111)$ present an ordered pattern of $\mathrm{O}$ vacancies, probably stabilized by the interaction with the substrate, which spatially modulates the oxygen vacancy formation energy within the coincidence cell. A single layer of cerium oxide grown on a $\mathrm{Cu}(111)$ substrate was reported to form a $(2 \times$ 2 ) array of interfacial oxygen vacancies ${ }^{[14]}$ and on the same substrate, using a specific growth procedure, a metastable cubic $\mathrm{Ce}_{2} \mathrm{O}_{3}$ phase in the form of a thin film has been stabilized. ${ }^{[15]}$ For 1 monolayer (ML) thick cerium oxide films on $\mathrm{Pt}(111)$ density functional theory (DFT) calculations identified different local interfacial bond lengths and a significant charge redistribution between the substrate $\mathrm{Pt}$ atoms and the $\mathrm{Ce}$ and $\mathrm{O}$ atoms of the oxide overlayer. ${ }^{[16]}$

Our previous studies of cerium oxide ultrathin epitaxial films on Pt(111) surfaces evidenced the presence of platinum oxide islands at the interface, which could mediate the epitaxial growth of the cerium oxide layer. ${ }^{[12]}$ Furthermore, we determined that ultrathin $\mathrm{CeO}_{2}$ layers have the fluorite structure with an in-plane contraction, ascribed to the formation of a 3:4 coincidence lattice with the substrate-i.e., to the matching of three oxide surface unit cells and four Pt surface unit cells, and that the contraction is released in thicker films. ${ }^{[17]}$ After vacuum thermal treatments, we observed the formation of highly reduced phases with peculiar surface reconstructions, possibly also stabilized by the presence of the Pt substrate, and we showed that the highly reduced phases can be easily reoxidized. ${ }^{[18]}$

In this work we report a study of the structure and charge configuration of the interface between cerium oxide and platinum, performed by aberration-corrected scanning transmission electron microscopy (STEM) and electron energy loss spectroscopy (EELS), combined with DFT+U calculations. The combination of high-resolution STEM measurements, to get insight into the atomic scale structure and chemistry of a buried interface-not easily accessible with other techniques, with a correct modeling of the system allowed us to clarify important aspects that determine the nature of the interaction between the two materials. In particular this work shows that a definitethough locally variable-registering is established between the oxide and the metal, it determines the preferential absorption geometry for the oxide and it explains why a significant fraction of interfacial cerium ions are reduced.

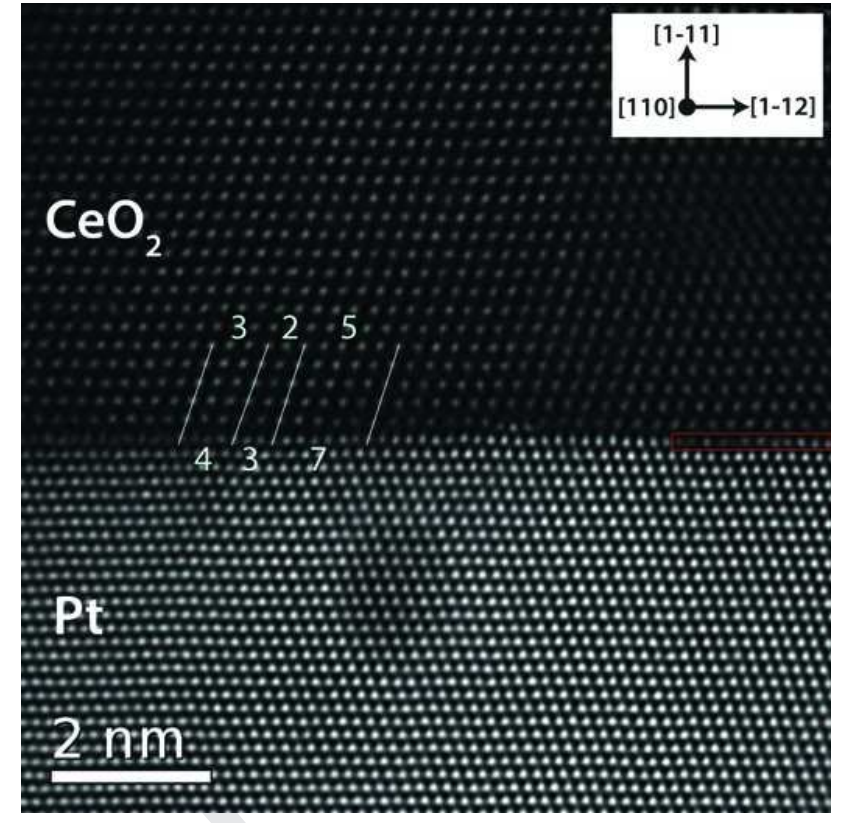

Figure 1. Cross sectional HAADF image of the $\mathrm{CeO}_{2} / \mathrm{Pt}(111)$ interface along the [110] zone axis. The blue lines indicate the interface coincidence registry that varies locally from $3: 4$ to $2: 3$ and 5:7. The red rectangle indicates an interfacial region with the periodicity of $\mathrm{Pt}$ atomic rows and a lower contrast, comparable to the one in the $\mathrm{CeO}_{2}$ layer.

\section{Results}

Figure 1 shows a representative high-angle annular dark-field (HAADF) image of the interface along the [110] zone axis. The low density of defects and the sharpness of the bright features in the $\mathrm{CeO}_{2}$ region is an indication of the good structural quality of the film. The symmetry and interatomic distances are compatible with the bulk fluorite structure in the (111) growth orientation. Different in-plane orientations between the substrate and the overlayer, ascribed to cerium oxide (111) domains rotated by $180^{\circ}$ with respect to each other, have been detected in different areas of the interface (see Figure S1, Supporting Information). The atomic scale details of the interface can be clearly detected and the interfacial registry between cerium oxide and platinum can be precisely determined. Some coincidence cells are highlighted by the blue lines in Figure 1. A statistical analysis of the sizes of the coincidence cells showed that the registering changes locally from $3: 4$ to $2: 3$ and 5:7 without a preferential coincidence cell size. The three configurations imply different in-plane local strain for the cerium oxide interfacial layer, namely, a 3.3\% compressive strain for the $3: 4$ coincidence, an $8.8 \%$ and a $1.5 \%$ expansive strain for the 2:3 and 5:7 coincidence structures, respectively.

In some areas of the sample, such as the one enclosed by a red rectangle in Figure 1, it is possible to identify an interfacial layer that has the periodicity of the atoms of the Pt substrate and a much lower contrast, closer to the one showed by the columns of the cerium oxide layer. Such areas extend up 
images

a) zone axis zone axis

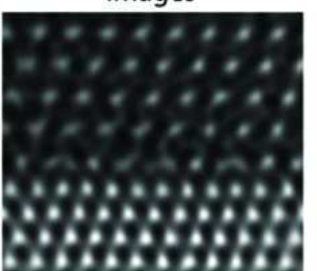

b)
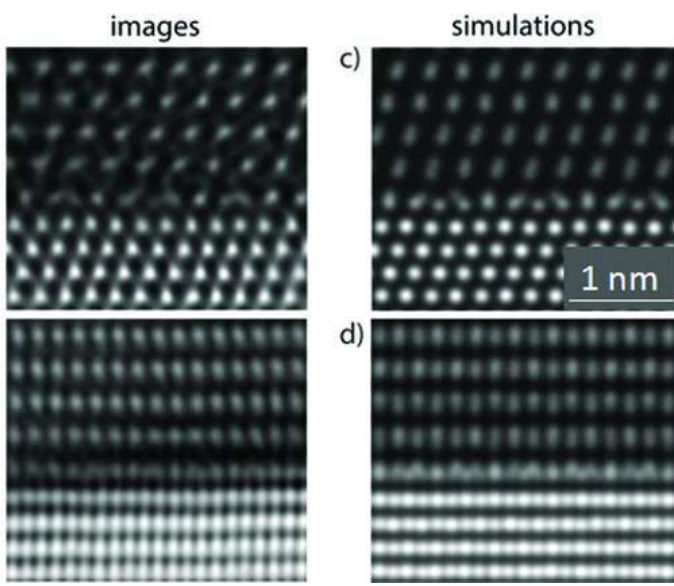

Figure 2. Cross sectional HAADF images of the $\mathrm{CeO}_{2} / \mathrm{Pt}(111)$ interface: a) along the [110] zone axis and b) along the [112] zone axis. Simulated images, obtained using the atomic positions of the model after structural relaxation, are shown in (c) and (d). An interfacial layer with the same periodicity as Pt and a much lower contrast can be identified.

to $\approx 10 \mathrm{~nm}$ and they are typically separated by several tens of nanometers. To have more information on the origin of the different interfacial contrast HAADF images were acquired along the [110] and [112] zone axes in regions in which the different contrast is very evident and compared by simulated HAADF images obtained using the atomic positions of the model after structural relaxation (Figure 2). A good agreement in terms of contrast and periodicity of the interface layer was obtained assuming a model in which the interfacial layer contains both $\mathrm{CeO}_{2}$ and $\mathrm{Pt}$ in roughly the same percentage. More precisely, the contrast modification originates from the presence of $\mathrm{CeO}_{2}$ and Pt stacked along the view direction, and it is ascribed to the formation of platinum oxide islands of single layer thickness on the Pt surface, already observed in ref. ${ }^{[12]}$. Since the intensity of each interfacial spot is related to the number of Pt atoms along the [110] direction, ${ }^{[19]}$ information on the 2D size and on the shape of the interfacial islands can be obtained from the spot intensity profile along the interfacial layer, as shown in Figure 3. A similar approach has shown to give reliable results also for the reconstruction of the 3D shape of nanoparticles. ${ }^{[20-23]}$ If we assume for example an approximate mirror symmetry of the island, we are able to extract the 2D shape of the island. An example of an interfacial island constructed using this method is shown in Figure 3c. Its shape and size are well compatible with the islands observed by STM and ascribed to $\mathrm{PtO}_{2}$ formation, which mediates the $\mathrm{CeO}_{2} / \mathrm{Pt}(111)$ epitaxy. ${ }^{[12]}$

To have more insight into the interaction between $\mathrm{CeO}_{2}$ and $\mathrm{Pt}$ it is very important to determine the preferential adsorption geometry. To this aim we have calculated the relaxed atomic structures in the three different interface configurations as shown in Figure 4, $\mathrm{O}_{\mathrm{i}}$-top, Ce-top, and $\mathrm{O}_{\mathrm{s}}$-top (see the Experimental Section), assuming the 3:4 interface coincidence. Although the differences in energy of the three configurations after structural relaxation are quite small, the $\mathrm{O}_{\mathrm{i}}$-top configuration results in slightly more stable than the Ce-top and the

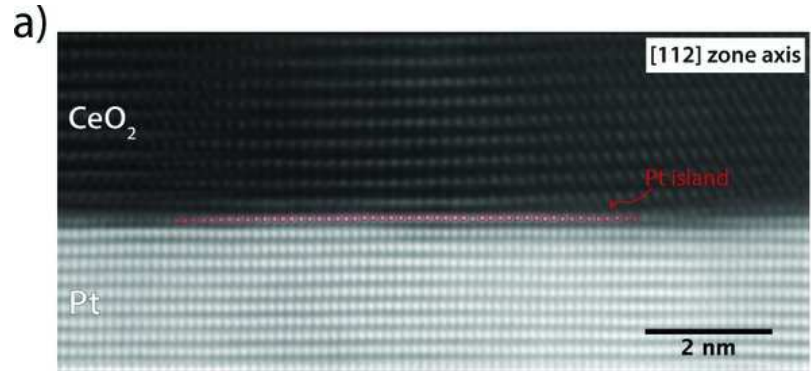

b)
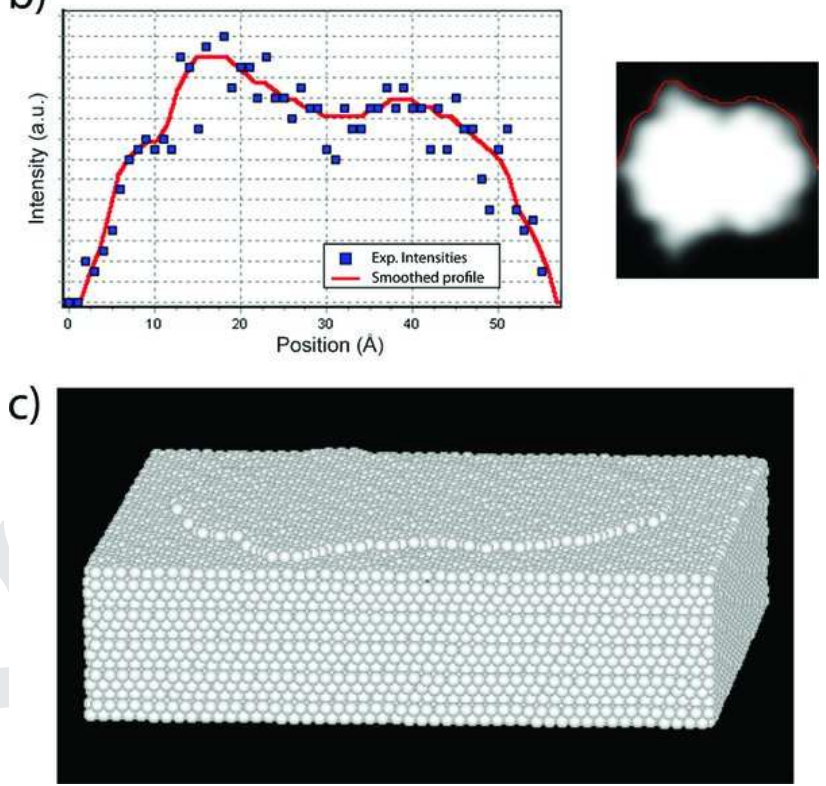

Figure 3. a) HAADF image of the $\mathrm{CeO}_{2} / \mathrm{Pt}$ interface along the [112] zone axis. b) Left: intensity profile of the interfacial spots (dots) and smoothed intensity profile (line); right: island shape obtained from the smoothed intensity profile assuming a mirror symmetry. c) 3D view of the interfacial island obtained by STEM_CELL using the smoothed spot intensity profile.

$\mathrm{O}_{\mathrm{s}}$-top $\left(\Delta E_{\text {(Ce-top)-(Oi-top) }}=9.1 \mathrm{meV}, \Delta E_{\text {(Os-top)-(Oi-top) }}=22.8 \mathrm{meV}\right.$ per interfacial $\mathrm{CeO}_{2}$ unit). The higher stability of the $\mathrm{O}_{\mathrm{i}}$-top configuration can be explained by considering the bonds between $\mathrm{Pt}$ and $\mathrm{O}$ atoms at the interface. The three configurations differ in the average length of interfacial Pt-O bonds. In particular, the number of Pt-O bonds shorter than $2.2 \AA$ within the supercell is 7, 6, and 4 for the $\mathrm{O}_{\mathrm{i}}$-top, Ce-top, and $\mathrm{O}_{\mathrm{s}}$-top configurations, respectively. Therefore, the stability of the $\mathrm{O}_{\mathrm{i}}$ top configuration is linked to the stronger bonding between $\mathrm{Pt}$ and $\mathrm{O}$ atoms at the interface. The shortest $\mathrm{O}$ bonds are obtained through a rumpling of $0.3 \AA$ of the interfacial Pt atoms toward the cerium oxide layer. The distance between the average position of $\mathrm{Pt}$ atoms in the topmost substrate plane and the average position of Ce atoms in the interfacial layer is comparable (and amounts to 3.07-3.10 $\AA$ ) for the three configurations (Figure 5). Notice that dispersion forces are not included in the calculations and that for this reason these distances can be 


\section{ADVANCED MATERIALS INTERFACES}

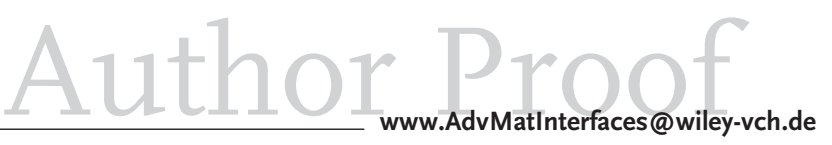

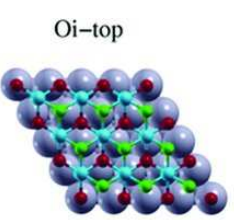
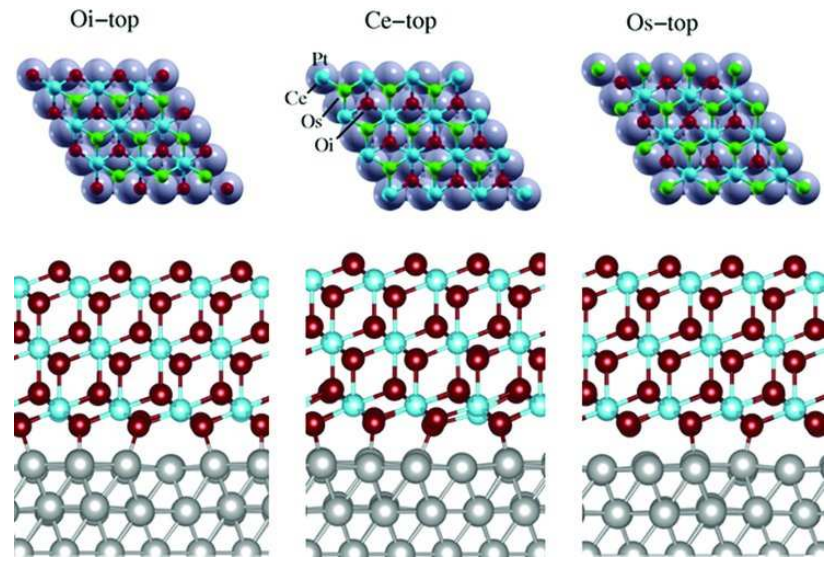

Figure 4. Top: top view of the three different interfacial configurations assumed in the calculations. Only a single Pt layer and the interfacial O-Ce-O trilayer are shown. The $\mathrm{Pt}$ atoms are sketched in gray, the interfacial $\mathrm{O}$ atoms in red, the $\mathrm{Ce}$ atoms in blue, and the $\mathrm{O}$ atoms in the third atomic layer in green. Bottom: side view of the three configurations shown in the top part.

slightly overestimated. The Ce-Ce interplanar distance in the oxide film is slightly increased compared to the interfacial layer (Figure 5). These values have been compared with the value of the lattice strain perpendicular to the interface, calculated experimentally from the HAADF-STEM images (Figure 5). The interplanar distance along the growth direction, measured on five different images, shows a substantial increase at the interface and a slight decrease in the cerium oxide film (Figure 5). The agreement between the calculated and experimental interplanar distances is good within the oxide film, where the values obtained from the experimental images are compatible with the ones calculated by theory, and with the value calculated for an unsupported $6 \mathrm{ML}$ oxide film (Figure 5). At the interface the slight discrepancy between the theoretical and experimental distances can be ascribed to the fact that the model considers only the 3:4 coincidence, while in the real system the epitaxy is more complex and the defects concentrate at the interface, together with the lack of dispersion forces in the DFT calculations.

To have more information on the interfacial configuration assumed by the cerium oxide film, we collected annular brightfield (ABF) images and HAADF images, which allow us to visualize light and heavy atoms in the structure ${ }^{[24,25]}$, and we compared them with image simulations using the atomic positions obtained from the calculations after structural relaxation. The simulated STEM images of the interface in the three geometric configurations are very similar if the HAADF imaging mode is used (see Figure S2, Supporting Information). A typical ABF image obtained is shown in Figure 6a. The contrast has been inverted to make it more similar to HAADF mode where atomic columns appear bright. In the cerium oxide layer the spots are more structured than in the images acquired in the HAADF mode, very likely due to scattering from $\mathrm{O}$ atoms, although, since the image formation process in the ABF mode

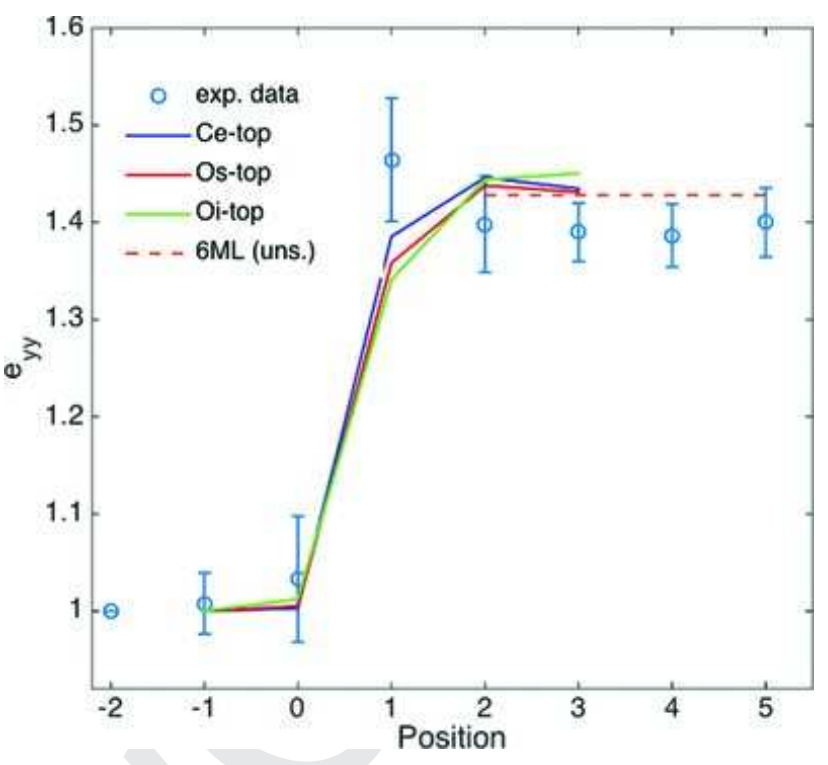

Figure 5. Strain perpendicular to the interface evaluated from HAADF images (blue dots) and from the theoretical model in the three interfacial configurations (solid lines). The strain is normalized to the Pt interplanar distance and plotted as a function of the number of layers ( 0 represents the position of the last Pt layer). The red dashed line indicates the value of the interatomic distance calculated for an unsupported $6 \mathrm{ML}$ cerium oxide film.

is quite complex, the observed features cannot be simply and directly related to the position of the oxygen columns. The simulations show indeed that the peaks in inverted ABF images are related to both $\mathrm{Ce}$ and $\mathrm{O}$ position in $\mathrm{CeO}_{2}$. In particular, in the experimental $\mathrm{ABF}$ image the interfacial layer shows periodic triangular symmetric features (marked by the white ellipses in Figure 6a). At the interface, where a superposition of $\mathrm{Pt}, \mathrm{O}$, and Ce atom columns is present, it is even more difficult to univocally attribute a feature in the image to a single atomic species (see Figure S3, Supporting Information), however the observed features can be compared to the simulated images. In fact, this image mode is affected by strong contrast change with parameters as thickness and defocus, and requires a work of extensive simulation for correct image interpretation. We found however a large region of parameters where the main contrast features appeared very similar to the experimental image. To properly compare the experimental and simulated images, 3:4 coincidence regions have been highlighted in the experimental image by yellow lines. The ABF simulated images show symmetric interfacial features for the $\mathrm{O}_{\mathrm{i}}$-top and $\mathrm{O}_{\mathrm{s}}$-top configurations (white ellipses in Figure $6 \mathrm{c}, \mathrm{d}$ ), while for the Ce-top configuration a clear asymmetric feature is observed (red ellipse in Figure 6b). Based on this comparison, we can exclude the Ce-top configuration from the possible interfacial configurations assumed by the system.

The calculations also evidence no net charge at the interface for these configurations. The small computed negative charge on the Pt substrate amounts to -0.15 e per unit cell, resulting 
a)

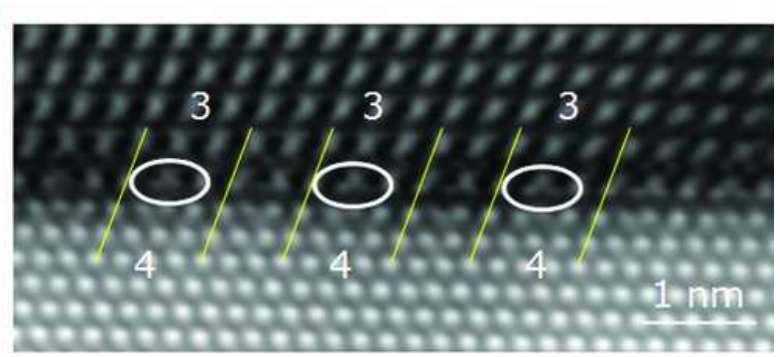

b)

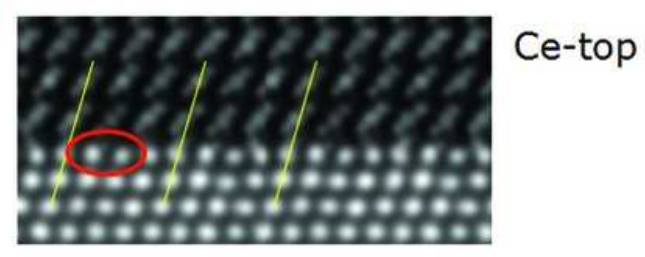

c)

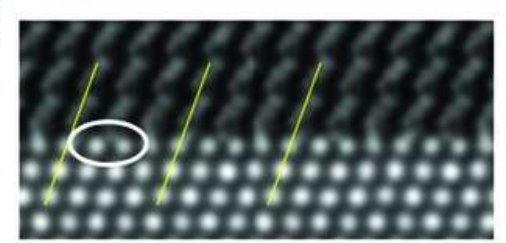

d)

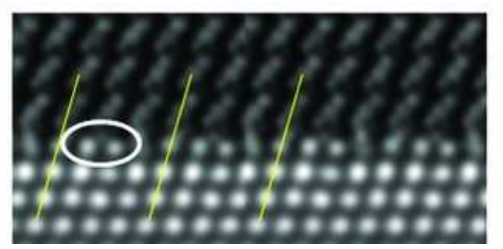

Figure 6. a) Inverted ABF-STEM image of the $\mathrm{CeO}_{2} / \mathrm{Pt}(111)$ interface measured along the [110] zone axis and simulated images in b) the (e-top, c) the $\mathrm{O}_{\mathrm{i}}$-top, and d) the $\mathrm{O}_{\mathrm{s}}$-top configurations. 3:4 coincidence areas are evidenced by yellow lines in the experimental and simulated images. The white ellipse in (a), (c), and (d) highlights a symmetric interfacial feature, while the red one in (b) highlights an asymmetric feature.

in a small positive charge in the oxide $\left(+0.02 \mathrm{e} / \mathrm{CeO}_{2}\right)$. Notably, we observed a modulated charge redistribution, in particular in the Pt interfacial layer. The Pt atoms involved in the shortest bonds with $\mathrm{O}$ are partly oxidized and show a positive charge of +0.10 e per atom, while the other Pt atoms carry a negative charge of -0.13 e per atom. Sizeable charge redistribution also occurs inside the oxide film. Indeed, the interfacial oxygen atoms are less reduced $(\Delta q=+0.06 \mathrm{e} / \mathrm{O})$, while the interfacial Ce atoms get a transferred charge of $\Delta q=-0.04$ e, resulting in an average negative charging of the interfacial Ce layer of $1.7 \%$. These calculations, which neglect the effect of the temperature, demonstrate that no spontaneous formation of $\mathrm{Ce}^{3+}$ takes place at the interface at low temperature. However, they do not exclude that other configurations containing $\mathrm{Ce}^{3+}$ could also be stable. To explore this possibility, we have recomputed the $\mathrm{O}_{\mathrm{i}}$-top configuration starting from a film structure with one of the nine Ce ions in the interfacial layer reduced to the

$3+$ state. This corresponds to an $11 \%$ average reduction of Ce ions in the interfacial layer. After the geometry optimization, this configuration maintains one electron in the Ce $4 f$ states as demonstrated by the spin density (Figure S4, Supporting Information). Interestingly, this structure is essentially isoenergetic with the $\mathrm{O}_{\mathrm{i}}$-top configuration (within $10 \mathrm{meV}$ ), although a barrier separates the two. While further studies are needed to explore the stability of different reduced configurations and to evaluate the barrier, this result proves that, if the system has enough energy to overcome the barrier, a fraction of $\mathrm{Ce}^{3+}$ ions can be present at the interface with Pt.

Information on possible local modifications of cerium oxidation state at different distances from the interface was obtained by collecting STEM-EELS spectrum images at the Ce $\mathrm{M}_{4,5}$ edge. Figure $7 \mathrm{a}, \mathrm{b}$ shows the HAADF signal and the corresponding Ce/Pt chemical map. In particular, the scale from green to red indicates the increasing amount of Ce evaluated from the total intensity of the $\mathrm{M}$ lines. In Figure $7 \mathrm{c}$ we show the $\mathrm{Ce}^{3+}$ concentration map obtained by fitting the local $\mathrm{M}_{4,5}$ edge with reference spectra from samples containing Ce only in 3+ $\left(\mathrm{CeF}_{3}\right)$ and $4+\left(\mathrm{CeO}_{2}\right)$ oxidation states, obtained from Turner et al. ${ }^{[26]}$ A non-negligible $\mathrm{Ce}^{3+}$ concentration is observed at the interface layer, and it amounts to an average value of $15 \% \pm 4 \%$. Figure $7 d$ shows two representative Ce $\mathrm{M}_{4,5}$ edge EELS spectra acquired at the interface and in the film bulk, respectively. The interface spectrum (red line) is clearly shifted toward lower energy loss and has a higher $\mathrm{M}_{5}$ to $\mathrm{M}_{4}$ branching ratio compared to the bulk one (blue line), as expected when the contribution from $\mathrm{Ce}^{3+}$ ions has a more relevant weight. ${ }^{[26]}$

\section{Discussion and Conclusions}

This work demonstrates the potential of STEM-based imaging and spectroscopic techniques, combined with DFT $+U$ calculations, for the study of interfaces between two materials. This is particularly important in systems as the one investigated here, in which the atomic scale details of the interface are expected to determine the properties of the combined material in the applications. For the specific $\mathrm{CeO}_{2} / \mathrm{Pt}$ case, a complete picture of the interface may help for example in designing systems that contain only (or mainly) atoms in the active phases, thus minimizing the amount of expensive noble metal employed in the catalysts.

The investigation of a model epitaxial system has allowed us to obtain a clear atomic scale picture of the interface between cerium oxide and platinum, and it has helped us to clarify several aspects of the interaction between the two materials. The calculations have given information on the relative stability of the different configurations and determined the relaxed atomic positions, used as an input for a proper comparison between measurements and simulated images. The sensitivity of $\mathrm{ABF}$ imaging mode also to lighter elements has been effective in discriminating the interfacial geometry assumed by the system. The theoretical calculations in turn have shown that the determinant parameter for the higher stability of the $\mathrm{O}_{\mathrm{i}}$-top configuration is the higher number of short bonds between $\mathrm{Pt}$ and $\mathrm{O}$ atoms. At variance with the result obtained in this study, 


\section{ADYANCED INTERFACES}

a)

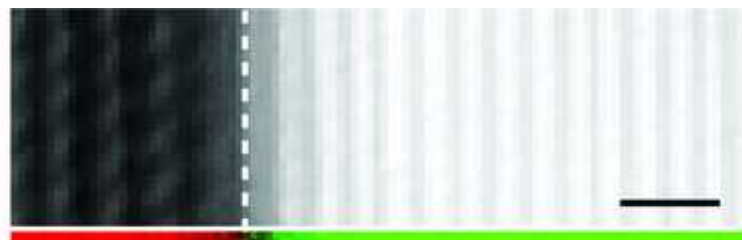

b)

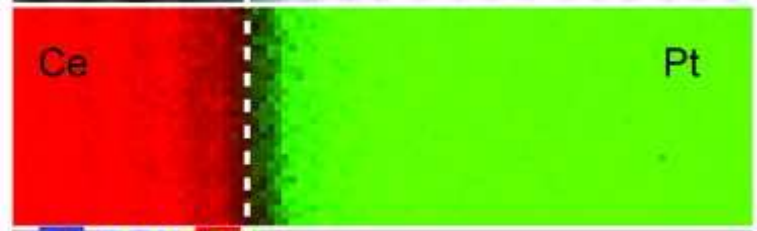

c)

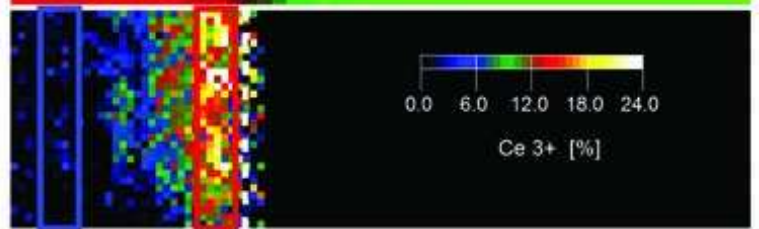

d)

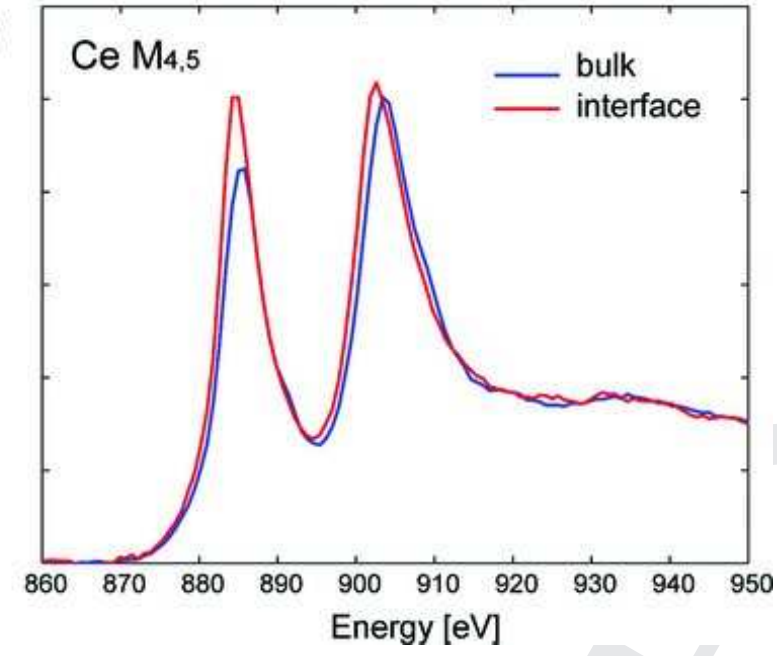

Figure 7. a) $\mathrm{HAADF}$ image of the $\mathrm{CeO}_{2} / \mathrm{Pt}(111)$ interface; b) $\mathrm{Ce} / \mathrm{Pt}$ chemical map obtained by STEM-EELS; c) map of $\mathrm{Ce}^{3+}$ concentration at the interface obtained by fitting the $\mathrm{Ce} \mathrm{M}_{4,5}$ edge spectra with spectra from reference samples containing $\mathrm{Ce}$ only in the $3+\left(\mathrm{CeF}_{3}\right)$ and $4+\left(\mathrm{CeO}_{2}\right)$ oxidation states; d) $\mathrm{Ce}$ $M_{4,5}$ edge spectra acquired at the interface (red line, average of the spectra measured in the area enclosed by the red rectangle in (c)) and in the $\mathrm{CeO}_{2}$ film bulk (blue line, average of the spectra measured in the area enclosed by the blue rectangle in (c)).

Spiel et al. in a theoretical study on a single cerium oxide ML on $\mathrm{Pt}(111)$ found the $\mathrm{O}_{\mathrm{s}}$-top interfacial configuration to be the most stable. ${ }^{[16]}$ However, in the case of a single cerium oxide layer, both the interface and the surface determine important modifications of the atomic positions, which give origin to a 2D system with a structure which is very different from the bulk or from thicker films.

Our previous studies showed that $2 \mathrm{ML}$ thickness films have the fluorite structure with an in-plane structural compression of a few percent, compatible with the 3:4 coincidence between $\mathrm{CeO}_{2}$ and $\mathrm{Pt}$, which is released when the thickness of the cerium oxide film is increased to $10 \mathrm{ML} \cdot{ }^{[17]}$ This work has demonstrated that a local epitaxial relation is maintained also when the film is several tens of atomic layers thick, although the strain relaxation implies the formation of areas that show also 2:3 and 5:7 local interfacial registries.

The evaluation of the interface charge configuration is certainly a crucial aspect to correctly describe the properties of the combined cerium oxide/Pt system. In general, the presence of $\mathrm{Ce}^{3+}$ ions in cerium oxide can be related to the formation of oxygen vacancies and/or to charge transfer from neighboring metal phases. In the present case of a buried interface between a metal support and a cerium oxide film, grown in highly oxidizing conditions, we considered the formation of oxygen vacancies rather unlikely and we examined the charge configuration of the interface between the Pt surface and the stoichiometric film. On model systems made of Pt nanoparticles supported on $\mathrm{CeO}_{2}$, Vayssilov et al. found that the formation of $\mathrm{Ce}^{3+}$ ions is induced on the oxide, and they ascribed it to a charge transfer from the metal nanoparticles to the support. ${ }^{[27]}$ This charge transfer is very relevant for the formation of the catalytically active Pt-O sites, which enhance the performance of the $\mathrm{Pt} / \mathrm{CeO}_{2}$ system. ${ }^{[28]}$ The calculations exposed in this work help us to get more insight into the factors that determine the charge transfer from Pt to cerium oxide. In the epitaxial system with 3:4 coincidence, a modulated redistribution of charge is observed at the epitaxial interface, without a net charge transfer. Indeed, the formation of polar Pt-O bonds at the interface produces a positive charge on the involved Pt atoms. On the other hand, the other interfacial Pt atoms bear a negative charge such that the two effects compensate. However the calculations also demonstrate that the reduction of a fraction of interfacial Ce is possible, although the formation of localized interfacial $\mathrm{Ce}^{3+}$ ions implies an energy barrier.

The metal work function has been shown to determine the charge transfer at the interface between ultrathin oxide films supported on single crystalline metal surfaces. ${ }^{[29-31]}$ Therefore, the larger charge transfer reported between $\mathrm{CeO}_{2}$ films and other metal supports, like $\mathrm{Cu}(111),{ }^{[14,31-33]} \mathrm{Ag}(111),{ }^{[31]}$ or $\mathrm{Au}(111)^{[31]}$, can be explained by considering the lower work function of $\mathrm{Cu}, \mathrm{Ag}$, and $\mathrm{Au}$ compared to Pt. Moreover, the value of the structural mismatch between the oxide and the metal has been suggested to play a role in the stability of $\mathrm{Ce}^{3+}$ sites at the interface. ${ }^{[31]}$ Being the mismatch larger in the case of $\mathrm{CeO}_{2} / \mathrm{Pt}(111)$ than in the case of $\mathrm{CeO}_{2} / \mathrm{Cu}(111)$, this might also explain the higher degree of reduction observed in cerium oxide films on $\mathrm{Cu}(111)$ compared to $\mathrm{Pt}(111)$. The differences in charge configuration at the $\mathrm{CeO}_{2} / \mathrm{Pt}(111)$ interface and in $\mathrm{Pt}$ nanoparticles on $\mathrm{CeO}_{2}{ }^{[27]}$ allow us to further infer that the specific electronic and atomic structures of very small particles are essential in promoting the $\mathrm{Pt}-\mathrm{CeO}_{2}$ charge transfer.

A non-negligible charge transfer from supported $\mathrm{Ag}$ nanoparticles and Ag layers to cerium oxide films has also been observed to take place. ${ }^{[34]}$ Since the cerium oxide films here investigated are covered by a thick Ag layer, we have considered the possibility of charge hopping from the $\mathrm{Ag} / \mathrm{CeO}_{2}$ interface to the $\mathrm{CeO}_{2} / \mathrm{Pt}(111)$. The barrier for electron hopping in cerium oxide was measured ${ }^{[35]}$ and confirmed theoretically ${ }^{[36]}$ to be $\approx 0.4 \mathrm{eV}$. Though the value of this barrier would allow 
charge hopping at room temperature, a clear driving force for electron migration through a $7 \mathrm{~nm}$ cerium oxide film from the $\mathrm{Ag} / \mathrm{CeO}_{2}$ interface to the $\mathrm{CeO}_{2} / \mathrm{Pt}$ could not be identified.

The possibility that some degree of reduction of Ce ions can be induced by the high intensity and high energy electron beam used for the STEM-EELS measurements has indeed been considered. However, we believe that this effect does not contribute significantly to the reduction observed at the $\mathrm{CeO}_{2} / \mathrm{Pt}$ interface, since the oxidation state of Ce ions in the film bulk results in being mainly $4+$ across the scan. Furthermore, the presence of interfacial cerium ions evidenced experimentally is corroborated by the theory, which demonstrated that $\mathrm{Ce}^{3+}$ ions in cerium oxide in proximity with platinum are stable.

In this study the quality of the HAADF-STEM images allowed us also to obtain a tentative 3D picture of interfacial islands, compatible with the one observed by scanning tunneling microscopy in the case of ultrathin films. ${ }^{[12]}$ With two images in different directions it would have been possible to perform a discrete tomography reconstruction with a less stringent a priori assumption. However, the aim here was only to give a reasonable approximation of the island shape. The appearance of approximate facets demonstrates that the result is not far from the one expected with an explicit energy minimization constraint, and it confirms that the potential of STEM-based techniques can be extended far beyond the mere 2D imaging of interfacial cross sections.

In conclusion, this work reveals interesting aspects of the atomic scale structure of the interfaces between cerium oxide and platinum. Among these, the tendency for the atoms in cerium oxide to adopt precise-though locally differentmatching with interfacial platinum atoms, the stabilization of the adsorption geometry which maximizes the number of short bonds between platinum and oxygen and the formation of $\mathrm{Ce}^{3+}$ ions at the interface between the oxide and the metal. These aspects certainly determine the overall properties of the $\mathrm{CeO}_{2} / \mathrm{Pt}$ system, making this study of interest also for understanding related cerium oxide/metal systems.

\section{Q6 4. Experimental Section}

The interface investigated in this study was grown and characterized in an ultrahigh vacuum system equipped with facilities for substrate cleaning, growth by reactive molecular beam epitaxy, in situ X-ray photoelectron spectroscopy (XPS), and low-energy electron diffraction (LEED) analysis. An ultrapure Pt(111) single crystal was used as a substrate. It was cleaned by repeated cycles of sputtering $(1 \mu \mathrm{A}, 1 \mathrm{keV})$ and annealing $(1040 \mathrm{~K})$ until the intensity of surface contaminants was below the XPS detection limit. The cerium oxide film was grown by evaporation of metallic cerium (evaporation rate $R=0.3 \AA$ min $^{-1}$, measured by a quartz microbalance) in $\mathrm{O}_{2}$ atmosphere $\left(\mathrm{P}_{\mathrm{O} 2}=1 \times 10^{-7} \mathrm{mbar}\right)$, followed by annealing in the same oxygen partial pressure to optimize stoichiometry, structure, and surface morphology. ${ }^{[12]}$ The oxygen pressure used to grow the cerium oxide film was at least one order of magnitude higher than the pressure needed to have the $\mathrm{CeO}_{2}$ stoichiometry in the films. The nominal film thickness is $7.2 \mathrm{~nm}$, as estimated from the Ce evaporation rate and the deposition time, enough to prevent possible damage at the interface induced by the subsequent processing steps. The film stoichiometry was checked by XPS and the surface structure by LEED, which showed a $\mathrm{CeO}_{2}$ stoichiometry and a high quality (111) surface orientation, respectively (see Figures S5 and S6, Supporting Information). The film was capped by a Ag layer with a nominal thickness of $12.6 \mathrm{~nm}$, to protect it from damage during the subsequent treatment. A cross sectional lamella was cut out of the single crystal ex situ using a Dual Beam apparatus (FEI Strata DB235M) and the focused ion beam lift-out method, with the procedure outlined in detail in the Supporting Information.

Aberration-corrected STEM was performed in an FEI Titan operated at $300 \mathrm{keV}$ and equipped with a high-brightness field emission gun (X-FEG), a CETCOR probe corrector from CEOS, and a Tridiem Image Filter/Spectrometer 866 ERS from Gatan Inc. for EELS. HAADF and ABF imaging were carried out with a Fischione 3000 and an FEI annular dark field detector, respectively. For the HAADF detector the inner semi-angle used was $55 \mathrm{mrad}$ and the outer one was $300 \mathrm{mrad}$, while for the ABF detector 25-26 mrad outer and 12-13 mrad inner semi-angles were used.

The strain analysis of HAADF images was performed using the Peak Pair algorithm to calculate the local changes in the distances between atomic columns and to obtain strain maps along the direction perpendicular to the film plane. The Peak Pair analysis was carried out using the dedicated tool within STEM_CELL software package. ${ }^{[37]}$

Spatially resolved information on the oxidation state of cerium ions was obtained by STEM-EELS spectrum imaging. In particular, the local $\mathrm{Ce}^{3+}$ concentration was derived from the shape of the $M_{4,5}$ absorption edges, following ref. ${ }^{26]}$. The local EELS spectra were fit using a linear combination of the spectra of two reference samples containing Ce ions only in $3+\left(\mathrm{CeF}_{3}\right)$ and only in $4+\left(\mathrm{CeO}_{2}\right)$ oxidation state to obtain the local concentration of $\mathrm{Ce}^{3+}$ ions with respect to $\mathrm{Ce}^{4+}$. The two reference spectra were obtained from Turner et al. ${ }^{[26]}$ and convolved with a Gaussian peak with an FWHM of $0.7 \mathrm{eV}$, to take into account the experimental energy resolution.

$\mathrm{DFT}+\mathrm{U}$ calculations for $3 \mathrm{ML}$ thick $\mathrm{CeO}_{2}$ films on a $\mathrm{Pt}(111)$ surface were performed to provide additional information on the investigated system. One cerium oxide ML is defined here as an O-Ce-O trilayer. The DFT $+\mathrm{U}$ method $^{[38]}$ with PBE functional ${ }^{[39]}$ and a U-J value of $4 \mathrm{eV}$ applied to the $\mathrm{Ce} f$ states were used, ${ }^{[27]}$ as implemented in the VASP code. ${ }^{[40,41]}$ The $\mathbf{Q 7}$ electron-core interaction was treated by using the projector augmented wave method ${ }^{[42]}$ with a plane waves energy cutoff of $400 \mathrm{eV}$. Atomic charges were estimated with the Bader decomposition method. ${ }^{[43,44]}$ The chosen supercell was made of $3 \times 3 \mathrm{CeO}_{2}(111)$ surface unit cells superposed to $4 \times 4 \mathrm{Pt}(111)$ surface unit cell, sampled with a $2 \times 2 \times 1$ Monkhorst-Pack $k$-points mesh in the reciprocal space. The cerium oxide lattice in this configuration was compressed by $3.3 \%$. The Pt surface was represented by a four-layer slab, with the two bottom layers fixed, while the surface layers and the oxide film were fully relaxed until all atomic forces were lower than $0.01 \mathrm{eV} \AA^{-1}$. The oxide was adsorbed on one side of the slab. A vacuum layer of at least $10 \AA$ separated the replicated slab and a dipole 


\section{MPNANCED INTERFACES}

correction was applied. Three different interfacial geometries were considered as shown in Figure 4. The three configurations differed in the relative position of the film with respect to the substrate and were identified in the following depending on the atom of the interfacial O-Ce-O trilayer located on top of the Pt atom on the upper left of the surface cell: an interfacial $\mathrm{O}$ atom for the $\mathrm{O}_{\mathrm{i}}$-top configuration, a Ce atom for the Ce-top configuration, and an $\mathrm{O}$ atom in the third atomic layer for the $\mathrm{O}_{\mathrm{s}}$-top configuration. A configuration in which one of the Ce ions is reduced to $\mathrm{Ce}^{3+}$ was also obtained starting from a locally distorted structure, as detailed in the Supporting Information.

Based on the atomic positions obtained by DFT $+\mathrm{U}$ after structural relaxation $\mathrm{HAADF}$ and $\mathrm{ABF}$ simulations were performed using the STEM_CELL software. ${ }^{[45]}$

\section{Supporting Information}

Supporting Information is available from the Wiley Online Library or from the author.

\section{Acknowledgements}

The authors acknowledge support from the Italian MIUR through the FIRB Project RBAP115AYN "Oxides at the nanoscale: multifunctionality and applications" and from the COST Action CM1104 "Reducible oxide chemistry, structure and functions." The authors also acknowledge the LMA at INA (Universidad de Zaragoza), where the aberration-corrected STEM studies were conducted, and the support of the European Union, Seventh Framework Program, under a contract for an Integrated Infrastructure Initiative Reference 312483ESTEEM2.

Received: July 12, 2015 Revised: August 27, 2015 Published Online: MM DD, YYYY

[1] C. M. Y. Yeung, K. M. K. Yu, Q. J. Fu, D. Thompsett, M. I. Petch, S. C. Tsang, J. Am. Chem. Soc. 2005, 127, 18010.

[2] A. M. Venezia, G. Pantaleo, A. Longo, G. Di Carlo, M. P. Casaletto, F. L. Liotta, G. Deganello, J. Phys. Chem. B 2005, 109, 2821.

[3] M. Cargnello, J. J. Delgado Jaén, J. C. Hernández Garrido, K. Bakhmutsky, T. Montini, J. J. Calvino Gámez, R. J. Gorte, P. Fornasiero, Science 2012, 337, 713.

[4] J. C. Conesa, Surf. Sci. 1995, 339, 337.

[5] C. Hardacre, G. M. Roe, R. M. Lambert, Surf. Sci. 1995, 326, 1.

[6] D. R. Mullins, S. H. Overbury, D. R. Huntley, Surf. Sci. 1998, 409, 307.

[7] K.-D. Schierbaum, Surf. Sci. 1998, 399, 29.

[8] S. Eck, C. Castellarin-Cudia, S. Surnev, M. G. Ramsey, F. P. Netzer, Surf. Sci. 2002, 520, 173.

[9] E. L. Wilson, W. A. Brown, G. Thornton, Surf. Sci. 2006, 600, 2555.

[10] J.-L. Lu, H.-J. Gao, S. Shaikutdinov, H.-J. Freund, Surf. Sci. 2006, 600,5004 .
[11] V. Matolín, J. Libra, I. Matolínová, V. Nehasil, L. Sedláček, F. Šutara, Appl. Surf. Sci. 2007, 254, 153.

[12] P. Luches, F. Pagliuca, S. Valeri, J. Phys. Chem. C 2011, 115, 10718.

[13] C. Castellarin-Cudia, S. Surnev, G. Schneider, R. Podlucky, M. G. Ramsey, F. Netzer, Surf. Sci. 2004, 554, L120.

[14] L. Szabova', O. Stetsovych, F. Dvořa'k, M. Farnesi Camellone, S. Fabris, J. Mysliveček, V. Matolín, J. Phys. Chem. C 2012, 116, 6677.

[15] V. Stetsovych, F. Pagliuca, F. Dvořák, T. Duchoň, M. Vorokhta, M. Aulická, J. Lachnitt, S. Schernich, I. Matolínová, K. Veltruská, T. Skála, D. Mazur, J. Mysliveček, J. Libuda, V. Matolín, J. Phys. Chem. Lett. 2013, 4, 866.

[16] C. Spiel, P. Blaha, Y. Suchorski, K. Schwarz, G. Rupprechter, Phys. Rev. B 2011, 84, 045412.

[17] P. Luches, F. Pagliuca, S. Valeri, F. Boscherini, J. Phys. Chem. C 2013, 117, 1030.

[18] P. Luches, F. Pagliuca, S. Valeri, Phys. Chem. Chem. Phys. 2014, $16,18848$.

[19] J. M. LeBeau, S. D. Findlay, L. J. Allen, S. Stemmer, Nano Lett. 2011, 11, 310.

[20] L. Jones, K. E. MacArthur, V. T. Fauske, A. T. J. van Helvoort, P. D. Nellist, Nano Lett. 2014, 14, 6336.

[21] H. Katz-Boon, C. J. Rossouw, M. Weyland, A. M. Funston, P. Mulvaney, J. Etheridge, Nano Lett. 2011, 11, 273.

[22] S. Van Aert, K. J. Batenburg, M. D. Rossell, R. Erni, G. Van Tendeloo, Nature 2011, 470, 374.

[23] D. G. Stroppa, R. D. Righetto, L. A. Montoro, L. Houben, J. Barthel, M. A. L. Cordeiro, E. R. Leite, W. Weng, C. J. Kiely, A. J. Ramirez, Nanoscale Res. Lett. 2013, 8, 475.

[24] S. D. Findlay, N. Shibata, H. Sawada, E. Okunishi, Y. Kondo, T. Yamamoto, Y. Ikuhara, Appl. Phys. Lett. 2009, 95, 191913.

[25] R. Ishikawa, E. Okunishi, H. Sawada, Y. Kondo, F. Hosokawa, E. Abe, Nat. Mater. 2011, 10, 278.

[26] S. Turner, S. Lazar, B. Freitag, R. Egoavil, J. Veerbeeck, S. Put, Y. Strauven, G. Van Tendeloo, Nanoscale 2011, 3, 885.

[27] G. N. Vayssilov, Y. Lykhach, A. Migani, T. Staudt, G. P. Petrova, N. Tsud, T. Skala, A. Bruix, F. Illas, K. C. Prince, V. Matolin, K. M. Neyman, J. Libuda, Nat. Mater. 2011, 10, 310.

[28] Q. Fu, H. Saltsburg, M. Flytzani-Stephanopoulos, Science 2003, $301,935$.

[29] J. Goniakowski, C. Noguera, Phys. Rev. B 2009, 79, 155433.

[30] S. Prada, U. Martinez, G. Pacchioni, Phys. Rev. B 2008, 78 235423.

[31] J. Graciani, A. B. Vidal, J. A. Rodriguez, J. F. Sanz, J. Phys. Chem. C 2014, 118, 2693.

[32] F. Yang, J. Graciani, J. Evans, P. Liu, J. Hrbek, J. F. Sanz, J. A. Rodriguez, J. Am. Chem. Soc. 2011, 133, 3444.

[33] J. A. Rodriguez, J. Graciani, J. Evans, J. B. Park, F. Yang, D. Stacchiola, S. D. Senanayake, S. Ma, M. Pérez, P. Liu, J. F. Sanz, J. Hrbek, Angew. Chem. Int. Ed. 2009, 48, 8047.

[34] P. Luches, F. Pagliuca, S. Valeri, F. Illas, G. Preda, G. Pacchioni, J. Phys. Chem C 2012, 116, 1122.

[35] H. L. Tuller, A. S. Novick, J. Phys. Chem. Solids 1977, 38, 859.

[36] J. J. Plata, A. M. Marquez, J. Fdez. Sanz, J. Phys. Chem. C 2013, 117, 14502.

[37] V. Grillo, F. Rossi, Ultramicroscopy 2013, 125, 112.

[38] S. L. Dudarev, G. A. Botton, S. Y. Savrasov, C. J. Humphreys, A. P. Sutton, Phys. Rev. B 1998, 57, 1505

[39] J. P. Perdew, K. Burke, M. Ernzerhof, Phys. Rev. Lett. 1996, 77, 3875.

[40] G. Kresse, J. Hafner, Phys. Rev. B 1993, 47, R558.

[41] G. Kresse, J. Furthmüller, Phys. Rev. B 1996, 54, 11169.

[42] P. E. Blöchl, Phys. Rev. B 1994, 50, 17953. 
[43] R. F. W. Bader, Chem. Rev. 1991, 91, 983.

[44] W. Tang, E. Sanville, G. Henkelman, J. Phys.: Condens. Matter. 2009, 21, 084204.

[45] V. Grillo, E. Rotunno, Ultramicroscopy 2013, 125, 97. 
Q1 APT to AU: AU: Affiliation 4 has been set as two separate affiliations-4 and 5-and the subsequent affiliation has been renumbered accordingly. Please confirm whether it is OK.

Q2 APT to AU: AU: Please confirm whether the citation of Ref. (13) in the sentence 'For example, Castellarin-Cudia et al. ...' is correct as included.

Q3 APT to AU: AU: Please define 'STM' at the first occurrence in the text.

Q4 APT to AU: AU: Please confirm whether edits made to the sentence 'However, we believe that this effect does not ...' retain the intended meaning.

Q5 APT to AU: AU: The sentence 'Among these, the tendency for the atoms in cerium ...' seems to be incomplete. Please check.

Q6 APT to AU: AU: As per journal style, the 'Experimental and Theoretical Section' has been changed to the 'Experimental Section.' Please check. APT to AU: AU: Please define 'VASP' at the first occurrence in the text. 